\title{
Shifts in gut and vaginal microbiomes are associated with cancer recurrence time in women with ovarian cancer
}

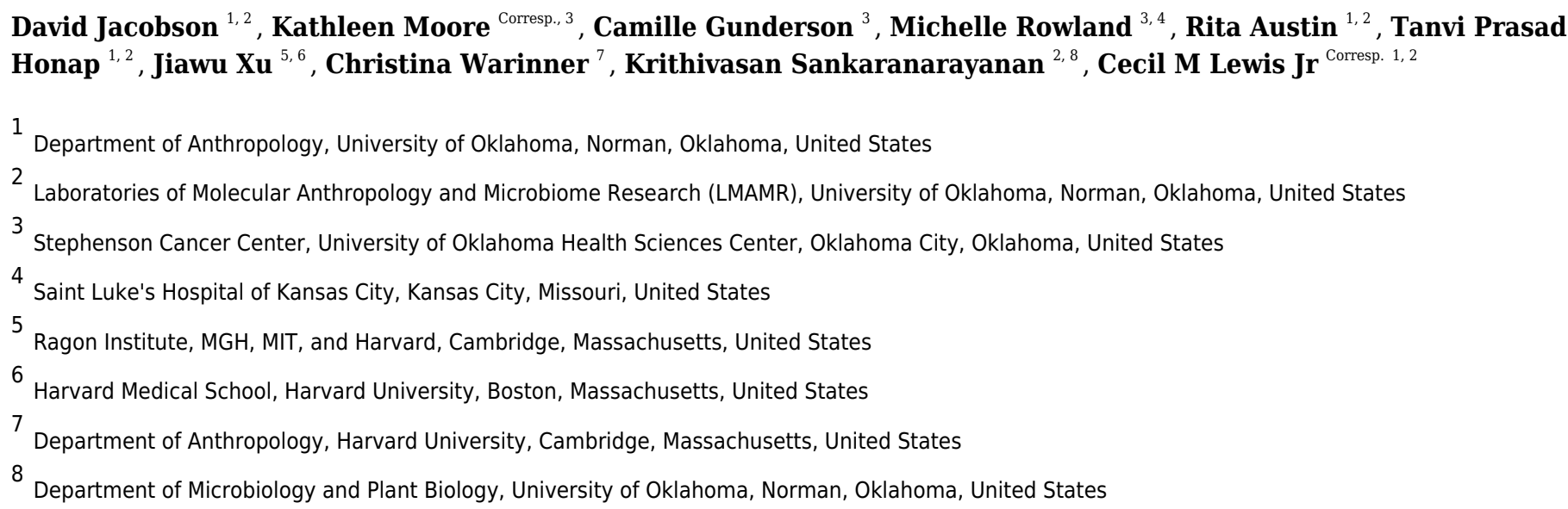

Many studies investigating the human microbiome-cancer interface have focused on the gut microbiome and gastrointestinal cancers. Outside of human papillomavirus driving cervical cancer, little is known about the relationship between the vaginal microbiome and other gynecological cancers, such as ovarian cancer. In this retrospective study, we investigated the relationship between ovarian cancer, platinum-free interval (PFI) length, and vaginal and gut microbiomes. We observed that Lactobacillus-dominated vaginal communities were less common in women with ovarian cancer, as compared to existing datasets of similarly aged women without cancer. Primary platinum-resistance (PPR) disease is strongly associated with survivability under one year, and we found over onethird of patients with PPR (PFI $<6$ months, $n=17$ ) to have a vaginal microbiome dominated by Escherichia ( $>20 \%$ relative abundance), while only one platinum super-sensitive (PFI>24 months, $\mathrm{n}=23$ ) patient had an Escherichia-dominated microbiome. Additionally, L. iners was associated with little, or no, gross residual disease, while other Lactobacillus species were dominant in women with $>1 \mathrm{~cm}$ gross residual disease. In the gut microbiome, we found patients with PPR disease to have lower phylogenetic diversity than platinum-sensitive patients. The trends we observe in women with ovarian cancer and PPR disease, such as the absence of Lactobacillus and presence of Escherichia in the vaginal microbiome as well as low gut microbiome phylogenetic diversity have all been linked to other diseases and/or pro-inflammatory states, including bacterial vaginosis and autoimmune disorders. Future prospective studies are necessary to explore the 
translational potential and underlying mechanisms driving these associations. 
1 Shifts in Gut and Vaginal Microbiomes are Associated 2 with Cancer Recurrence Time in Women with Ovarian 3 Cancer

David K. Jacobson ${ }^{1,2}$, Kathleen N. Moore ${ }^{3}$, Camille C. Gunderson ${ }^{3}$, Michelle R. Rowland ${ }^{3,4}$, Rita M. Austin ${ }^{1,2}$, Tanvi P. Honap ${ }^{1,2}$, Jiawu Xu ${ }^{5,6}$, Christina G. Warinner ${ }^{7}$, Krithivasan Sankaranarayanan ${ }^{2,8}$, Cecil M. Lewis, $\mathrm{Jr}^{1,2}$

${ }^{1}$ Department of Anthropology, University of Oklahoma, Norman, OK, United States

${ }^{2}$ Laboratories of Molecular Anthropology and Microbiome Research, University of Oklahoma, Norman, OK, United States

${ }^{3}$ Stephenson Cancer Center, University of Oklahoma Health Sciences Center, Oklahoma City, OK, United States

${ }^{4}$ Saint Luke's Hospital of Kansas City, Kansas City, MO, United States

${ }^{5}$ Ragon Institute of MGH, MIT and Harvard, Massachusetts General Hospital, Cambridge, MA, United States

${ }^{6}$ Harvard Medical School, Boston, MA, United States

${ }^{7}$ Department of Anthropology, Harvard University, Cambridge, MA, United States

${ }^{8}$ Department of Microbiology and Plant Biology, University of Oklahoma, Norman, OK, United States

Co-Corresponding Authors

Cecil M. Lewis, $\mathrm{Jr}^{1,2}$

Stephenson Research and Technology Center

101 David L. Boren Blvd

Norman, OK 73019, USA

$+1(405) 325-3415$

cmlewis@ou.edu

Kathleen N. Moore ${ }^{3}$

Stephenson Cancer Center

$800 \mathrm{NE} 10^{\text {th }}$ Street

Oklahoma City, OK 73104, USA

+1 (405) $271-7770$

Kathleen-moore@ouhsc.edu 
42 Many studies investigating the human microbiome-cancer interface have focused on the gut 43 microbiome and gastrointestinal cancers. Outside of human papillomavirus driving cervical

44 cancer, little is known about the relationship between the vaginal microbiome and other 45 gynecological cancers, such as ovarian cancer. In this retrospective study, we investigated the 46 relationship between ovarian cancer, platinum-free interval (PFI) length, and vaginal and gut 47 microbiomes. We observed that Lactobacillus-dominated vaginal communities were less 48 common in women with ovarian cancer, as compared to existing datasets of similarly aged 49 women without cancer. Primary platinum-resistance (PPR) disease is strongly associated with 50 survivability under one year, and we found over one-third of patients with PPR (PFI $<6$ months, $n$ $51=17)$ to have a vaginal microbiome dominated by Escherichia ( $>20 \%$ relative abundance), 52 while only one platinum super-sensitive (PFI $>24$ months, $\mathrm{n}=23$ ) patient had an Escherichia53 dominated microbiome. Additionally, L. iners was associated with little, or no, gross residual 54 disease, while other Lactobacillus species were dominant in women with $>1 \mathrm{~cm}$ gross residual 55 disease. In the gut microbiome, we found patients with PPR disease to have lower phylogenetic 56 diversity than platinum-sensitive patients. The trends we observe in women with ovarian cancer 57 and PPR disease, such as the absence of Lactobacillus and presence of Escherichia in the vaginal 58 microbiome as well as low gut microbiome phylogenetic diversity have all been linked to other 59 diseases and/or pro-inflammatory states, including bacterial vaginosis and autoimmune 60 disorders. Future prospective studies are necessary to explore the translational potential and 61 underlying mechanisms driving these associations.

62

63

\section{Introduction}


65 Ovarian cancer is the most deadly gynecological cancer (1); it kills approximately 14,000 women

66 in the United States annually, accounting for $4.9 \%$ of all cancer-related deaths in females in the

67 United States. In the majority of cases $(>80 \%)$, ovarian cancer is not detected until stage III or

68 later, primarily due to the nonspecific nature of ovarian cancer symptoms and lack of informative

69 biomarkers (2, 3). Early-stage (I or II) detection results in substantially greater five-year

70 survivability compared to late-stage diagnosis (III or IV): $70 \%$ versus $36 \%$ survival rate,

71 respectively (4), highlighting the importance of discovering early-disease biomarkers.

72

73 The standard course of primary treatment in ovarian cancer is cytoreductive surgery (CRS) in

74 combination with platinum-based chemotherapy (5), which causes cytotoxicity through the

75 formation of intra- and inter- strand adducts on DNA in cancer cells (6). The diameter of the

76 remaining tumor after CRS, referred to as gross residual disease, is an important predictor of

77 patient outcome, as individuals with no residual disease or residual disease $<1 \mathrm{~cm}$ have

78 improved survivability compared to those with tumors $>1 \mathrm{~cm}$ after CRS (7). The combination of

79 CRS and platinum-based chemotherapy is highly effective with approximately $80 \%$ of all

80 patients showing no evidence of disease at the conclusion of initial therapy; however,

81 recurrences occur in $70-80 \%$ of advanced stage patients and $20-25 \%$ of early-stage patients (8).

82 Primary platinum resistance (recurrence of cancerous growth within six months of primary

83 treatment cessation) develops in about $20 \%$ of patients, and is highly problematic because it is

84 associated with a survivability of under one year and fewer effective treatment options (9). Other

85 patients may remain free of cancerous growth for more than two years, but the risk of recurrence

86 and eventual development of treatment-resistant cancer is still unacceptably high (10-13). This 
87 merits a focus on discovering biomarkers of ovarian cancer and drivers of platinum-resistance to

88 facilitate early cancer detection as well as better understand variation in treatment outcomes.

89

90 Recent evidence suggests that the human microbiome is an important factor in tumorigenesis,

91 carcinogenesis, and effectiveness of chemotherapy (14-17). Gut microbiome dysbiosis can

92 influence colorectal carcinogenesis via production of genotoxic metabolites, such as colibactin,

93 and through the promotion of a pro-inflammatory state, which contributes to cancer cell

94 proliferation, angiogenesis, and metastasis $(14,17-20)$. While most studies on the microbiome-

95 cancer relationship have focused on the gut microbiome, there is growing evidence in support of

96 the relationship between the vaginal microbiome and gynecological cancers. For example,

97 human papillomavirus (HPV) is a known causative agent of cervical cancer (21-23), while pelvic

98 inflammatory disease, which is associated with shifts in vaginal microbiome composition (24),

99 has been linked to ovarian cancer development (25). Yet, there are still many unknowns about

100 the relationship between the vaginal microbiome and gynecological cancers $(21-23,26)$.

101 Likewise, links between the vaginal microbiome and platinum-sensitivity remain elusive;

102 however, a previous study demonstrated that cancerous growths in mice with antibiotic-depleted

103 gut microbiomes were less susceptible to platinum-chemotherapies compared to those with high

104 gut microbiome diversity (15). The microbiome has a strong, bi-directional relationship with the

105 host immune system $(27,28)$ and immune cells in the mice with a depleted gut microbiome

106 produced fewer reactive oxygen species (ROS) than control mice (15). Decreased ROS

107 production and platinum-resistance in mice with low gut microbiome diversity suggests a role

108 for the microbiome in response to platinum-chemotherapy because ROS play a part in cell

109 apoptosis after exposure to platinum chemotherapeutic agents (29). 
111 In this study, we assessed how the vaginal and gut microbiomes vary in ovarian cancer patients

112 with different platinum-sensitivities, with the aim of determining whether the human microbiome

113 can be used as a biomarker of platinum-sensitivity.

114

\section{Materials \& Methods}

\section{Study Population}

117 Patients, who carried a diagnosis of advanced (Stage III/IV) epithelial ovarian cancer and who

118 were classified as primary platinum-resistant (platinum-free interval [PFI] from completion of

119 primary platinum-based chemotherapy $<6$ months) or platinum super-sensitive (PFI $>24$

120 months) and were being treated at the Stephenson Cancer Center at the University of Oklahoma

121 Health Sciences Center, were approached to participate in this study. Patients were approached

122 when they either developed primary platinum-resistant disease or when they were identified as

123 platinum super-sensitive. Patients who had already been diagnosed with primary platinum-

124 resistant disease or as platinum super-responders but had moved on to additional therapy, as well

125 as patients on active anti-cancer therapy or in surveillance, were also included in this study.

126 Patients were excluded if: 1) they were taking antibiotics at the time of sample collection or

127 within 14 days prior to sample collection, or 2) they had active vaginal bleeding or known

128 entero-vaginal fistulae. Samples were also collected from five individuals who were referred for

129 ovarian cancer treatment at the Stephenson Cancer Center but ultimately had benign tumors;

130 these served as a non-chemotherapy exposed control group. Sample collection periods and

131 treatment procedures are outlined in Fig. S1. A brief summary of demographic and medical

132 treatment history for participants in this study $(\mathrm{n}=45$, median age 62.2 , age range $33-83)$ is 
133 provided in Table 1. This study was approved by the University of Oklahoma Health Sciences

134 Center Institutional Review Board (February 22nd 2016 , reference \#6458) and all participants 135 gave written informed consent for their participation in the study.

137 All patients were treated initially with platinum and taxane chemotherapy for a planned six to 138 eight cycles. These regimens included paclitaxel and carboplatin given every 21 days, paclitaxel 139 given weekly with every $21^{\text {st }}$ day carboplatin, or intraperitoneal administration of either cisplatin

140 or carboplatin with intravenous and intraperitoneal paclitaxel. In patients with platinum resistant 141 disease, standard of care options after recurrence included pegylated liposomal doxorubicin 142 (PLD), weekly paclitaxel, gemcitabine, topotecan or bevacizumab given as monotherapy or in 143 combination therapy. Patients were also screened for eligibility for clinical trials. Patients with a 144 PFI $>24$ months had not recurred at the time of study participation and were followed every 6 145 months with surveillance of Ca-125 values and exams. For those who had recurred beyond 24 146 months, treatment options included several platinum-based doublets including carboplatin and 147 PLD given every 28 days, carboplatin and paclitaxel given every 21 days or carboplatin and 148 gemcitabine given on a day one and day eight or day one and day fifteen schedule. Each patient 149 completed a minimum of six cycles of treatment and could undergo more cycles as long as the 150 patient was responding and tolerating therapy.

151

\section{Sample Collection}

153 Samples were collected during standard of care exams in the gynecologic oncology clinic at the 154 Stephenson Cancer Center in Oklahoma City, OK. Catch-All Sample Collection Swabs

155 (Epicentre) were used to collect vaginal and fecal samples. Vaginal swabs were collected from 
156 three sites per individual: vaginal introitus (VIT), mid-vagina (MDV), and posterior fornix

157 (VPF), and then placed into a dry sample collection tube. Fecal samples were collected via a

158 rectal digital exam, after which any stool collected was placed on a Catch-All swab and placed in

159 a dry collection tube. Two swabs were collected from each site (bilaterally from the vaginal sites

160 and sequentially for the rectal samples). Each participant completed a quality-of-life survey

161 regarding their medical treatment history, antibiotic use within the past year, vitamin

162 consumption, socioeconomic status, and other lifestyle metadata (Table S1).

163

164 Laboratory Methods

165 DNA was extracted from the left-side vaginal swab and first fecal swab from each patient, using

166 the MoBio PowerSoil DNA Isolation Kit (now Qiagen DNeasy PowerSoil Kit), following

167 manufacturer's protocols with the addition of a ten-minute incubation at $65^{\circ} \mathrm{C}$ prior to the initial

168 bead-beating step, as recommended in the Manual of Protocols for the Human Microbiome

169 Project (30). A quantitative PCR (qPCR), using the SYBR Green PCR Master Mix (Applied

170 Biosystems) and primers targeting the V4 region of the bacterial 16S rRNA gene (31), was

171 conducted; dilutions of Escherichia coli DNA corresponding to known 16S rRNA gene copy

172 numbers were used as quantification standards for the DNA extracts. DNA extracts were

173 amplified in triplicate, using Phusion High-Fidelity DNA polymerase (ThermoFisher Scientific)

174 and Illumina-compatible primers 515F and 806R (targeting the V4 region of the16S rRNA gene)

175 with error-correcting Golay barcodes incorporated into the 806R reverse primer (31). PCR

176 products were pooled in equimolar concentrations, purified with the MinElute PCR purification

177 kit (Qiagen), then size-selected between 300 and 450 basepairs using a PippenPrep, quantified 
178 using KAPA Biosystems Illumina library quantification kit, and sequenced across multiple runs

179 of an Illumina MiSeq (500 cycles paired-end sequencing, v2 reagent kit).

180

181 Bioinformatic Methods

182 AdapterRemoval (v2) (32) was used to filter out reads with uncalled bases, reads with Phred 183 quality threshold $<30$, and reads less than $150 \mathrm{bp}$ in length. Quality filtered paired-end reads 184 were merged using AdapterRemoval (v2) (32) and then demultiplexed with QIIME (v1.9), 185 followed by removal of chimeric sequences and low-abundance ( $<5$ total sequences) reads (33). 186 The remaining sequences were used for de novo Operational Taxonomic Unit (OTU) clustering 187 with USEARCH (v10) at 97\% sequence similarity (34). Taxonomy was assigned to each OTU 188 representative using the EzBioCloud 16S rRNA gene database (35). The resulting OTU table 189 was rarefied to 9000 reads and downstream analysis was performed in QIIME (v1.9) (33). The 190 post-rarefaction sample breakdown was: PFI $>24(n=23), \operatorname{PFI}<6(n=17)$, and benign $(n=5)$. 191 Further details of the bioinformatic methods are given in the Supplementary Material.

\section{Statistical Methods}

194 Phylogenetic diversity and weighted/unweighted UniFrac (36) metrics were generated in QIIME 195 (v1.9) with FastTree2 (37). Tests for significance between study groups for alpha and beta 196 diversity were performed using Kruskal-Wallis and PERMANOVA tests, respectively, in R (38).

197 Vaginal samples were classified into clusters by the dominant bacterial taxon found in each 198 sample, as determined by Ward hierarchical clustering (39), and visualized as a heatmap using 199 the gplots package in R (40). If there were no dominant bacteria, the sample was classified as 200 diverse. Median-unbiased estimated odds ratios were calculated to determine whether study 
201 groups had significantly different odds of dominant bacteria; reported odds ratios and 95\%

202 confidence intervals were log-transformed. Kruskal-Wallis tests with a Benjamini and Hochberg

203 false-discovery rate adjustment were used to evaluate differential abundance of individual taxa

204 between study groups. Odds ratios were calculated using epitools (41) in R. Plots were generated 205 using the ggplot2 (42) and ColorBrewer (43) packages in R.

206

207 Results

208

\section{Vaginal microbiome}

209 Samples from the different vaginal sites (VIT, MDV, VIT) that originated from the same

210 individual showed similar taxonomic beta-diversity profiles (Fig. S2A-C). Sequencing failed for

211 at least one of the three vaginal sites of eleven individuals (Supplementary Material), which

212 presented difficulty in analyzing each vaginal site individually as the failed specimens were not

213 limited to only one vaginal site. Due to the similarity in composition between vaginal sites in

214 each individual and the relatively high number of samples which failed sequencing, we

215 concatenated sequencing reads from each individual's three vaginal samples into a single

216 representative vaginal microbiome sample per individual, and then performed downstream

217 analysis with this single representative sample, unless otherwise noted. Combining vaginal sites

218 for each individual allowed us to retain a sample size of 45. Firmicutes was the most dominant

219 phylum in the vaginal microbiome and it was found at over $50 \%$ relative abundance in $40 \%$ of

220 individuals (Fig. S3A), while Proteobacteria, Bacteroidetes, and Actinobacteria were the next

221 most abundant phyla and found at $>50 \%$ relative abundance in the vaginal microbiome of $13.3 \%$,

$22213.3 \%$, and 6.7\% of individuals, respectively. At the genus level, Lactobacillus, Prevotella, 
223 Escherichia, Gardnerella, and Streptococcus were the most dominant bacteria and accounted for

$22457.2 \%$ of all reads (Fig. S3B).

225

226 Individuals were grouped into the following study groups as outlined in the methods section

227 (Fig. S1): PFI < 6 Months $(\mathrm{n}=17)$, PFI $>24$ Months $(\mathrm{n}=23)$, and benign $(\mathrm{n}=5)$. Table 1

228 presents summary statistics for these study groups. Iterative cytoreductive surgery (iCRS) was

229 more common in PFI $<6$ months while primary cytoreductive surgery (pCRS) was more

230 common in PFI $>24$ months individuals (log Odds Ratio $[\mathrm{OR}]=3.02,95 \%$ CI $1.37-4.68$, p-

231 value $=0.0003$ ). As expected, PFI $<6$ months individuals underwent platinum chemotherapy

232 treatment more recently than PFI $>24$ months individuals $\left(\mathrm{p}\right.$-value $\left.=4.32 \times 10^{-7}\right)$ but no other

233 metadata variable was significantly associated with PFI status (Table S2).

234

235 Vaginal microbiome communities were clustered into five community-dominance groups using

236 Ward hierarchical clustering: Lactobacillus cluster, Escherichia cluster, Gardnerella cluster,

237 Prevotella cluster, and a high diversity cluster (Fig. 1). Results below are presented

238 demonstrating how each vaginal community (excluding Gardnerella due to small cluster size)

239 relate to clinical variables, $\mathrm{p}$-values for each vaginal community and clinical variable are given

240 in Table S3.

241

242 Dominance groups were evenly distributed between patients with PFI $<6$ months, PFI $>24$

243 months, and benign, with the exception of higher-than-expected dominance of Escherichia in

244 patients with PFI $<6$ months; five of the six Escherichia-dominated vaginal communities

245 identified with hierarchical clustering belonged to patients with PFI $<6$ months (Fig. 1). Vaginal 
246 microbiomes dominated by Escherichia had higher odds of occurring in PFI $<6$ months

247 compared to PFI $>24$ months $(\log \mathrm{OR}=2.812,95 \%$ CI $0.267-5.62$, p-value $=0.024$, Fig. $2 \mathrm{~A})$.

248 Additionally, one of the patients with PFI $<6$ months and one of the patients with benign

249 pathology identified with a 'diverse' vaginal microbiome had Escherichia at greater than $20 \%$

250 relative abundance, while no other patients with PFI $>24$ months had Escherichia relative

251 abundance above 5\% (Fig. 1). In total, 35.3\% of the patients with PFI $<6$ months had

252 Escherichia at greater than $20 \%$ relative abundance in the vaginal microbiome, compared to

$2534.34 \%$ of PFI $>24$ months and only one of five benign individuals. Even though iterative

254 cytoreductive surgery and Escherichia are more common in PFI $<6$ months individuals, we

255 found no significant relationship between iCRS and Escherichia abundance (Table S3, Fig. 2A,

256 p-value $=0.292$ ). Moreover, outside of the relationship between Escherichia and platinum

257 resistance, Escherichia showed no significant association with any of the other health or lifestyle

258 factors we examined (Table S3, Fig. 2A). Although Escherichia is a common lab-grown

259 bacterium and found in feces, our analysis demonstrates Escherichia abundance in the vaginal

260 samples is biological and not a technical artifact (Supplementary Material).

261

262 Approximately 24\% (11 of 45) of patients in this study had Lactobacillus-dominated

263 communities, which is significantly lower as compared to studies of similarly aged women

264 without ovarian cancer $(\mathrm{p}$-value $=0.037)(44,45)$. Other studies have found Lactobacillus to be

265 less abundant in Black and Hispanic women (46). and our study consisted of 39 women who

266 self-reported ethnicity as white, two self-reported as Black, and four self-reported as Native

267 American (Table 1). Each of the Black and Native American women had a non-Lactobacillus

268 dominated vaginal microbiome (Table S1) but ethnicity was not a statistically significant 
269 determinant of Lactobacillus-dominance (Fig 2B, $\log \mathrm{OR}=-1.63,95 \% \mathrm{CI}-4.62-1.30$, p-value

$270=0.27$ ). High microbial cell density, as gauged through qPCR with a standard curve generated

271 from controls with known cell density, was positively correlated with vaginal Lactobacillus-

272 dominance, although somewhat weakly $\left(\mathrm{R}^{2}=0.278\right.$, Fig. S4). Consumption of antibiotics within

273 the past month was associated with a lack of Lactobacillus-dominance (Fig. 2B); however, this

274 relationship was not significant $(\log \mathrm{OR}=-2.12,95 \% \mathrm{CI}-5.54-0.84, \mathrm{p}$-value $=0.0515)$.

275 Additionally, Lactobacillus abundance was uncommon in individuals likely to be estrogen

276 negative (post-menopause and not on hormone replacement therapy) but this result was also not

277 significant $(\log \mathrm{OR}=-1.32,95 \% \mathrm{CI}-2.83-0.33$, p-value $=0.0744)$. Lactobacillus-dominance

278 did not have a strong relationship with any of the other health or lifestyle factors we tested,

279 including PFI length (Table S3, Fig. 2B). Only 20\% (1 of 5) of patients with benign pathology

280 had a Lactobacillus-dominated microbiome but small sample size prohibits statistical inference.

281

282 Previous studies have indicated that different Lactobacillus species in the vaginal microbiome

283 may have different roles and differential influence on host biology (47-49). While we identified

284 L. iners, L. brevis, L. mucosae, L. reuteri, L. zeae, and L. delbrueckii in the vaginal microbiome,

$28599 \%$ of the Lactobacillus reads in our study were either unclassified at the species level or

286 mapped to L. iners (Table S4); ultimately there was not sufficient data to assess the relationship

287 between most of these Lactobacillus species and clinical variables. Nevertheless, in individuals

288 with high Lactobacillus abundance $(\mathrm{n}=11)$, we found L. iners at significantly higher abundance

289 in patients with either no gross residual disease or residual disease $<1 \mathrm{~cm}(\mathrm{n}=7)$, compared to

290 patients with residual disease $>1 \mathrm{~cm}(\mathrm{n}=4$, $\mathrm{p}$-value $=0.0359$, Fig. 3$)$. 
292 We did not observe significant associations between either Prevotella and clinical variables

293 (Table S3, Fig. 2C) or the high diversity community and clinical variables (Table S3, Fig. 2D).

294 However, Prevotella was less common in those with residual disease $>1 \mathrm{~cm}$ and those with a

295 history of hormonal disease (Fig. 2C), while a highly diverse vaginal microbiome was more

296 common in women over 60 years old and in patients with a history of hormonal disorders, such

297 as thyroid disease (Fig. 2D); but once again, none of these associations were statistically

298 significant (Table S3, p-value $>0.05$ ).

299

300 Gut microbiome

301 The gut microbiome was colonized by typical members of the gut microbiome at the phylum

302 (Bacteroidetes, Firmicutes, Proteobacteria) and genus (Bacteroides, Akkermansia,

303 Faecalibacterium, Ruminococcus, and Prevotella) levels (Fig. S5A-B). The PFI > 24 months and

$304 \mathrm{PFI}<6$ months groups were not significantly different with respect to fecal unweighted and

305 weighted UniFrac beta diversity distances as tested through PERMANOVA (Fig. 4, Fig. S6A-

306 C); however, similar to the vaginal microbiome samples, there was a small subset of individuals

$307(\mathrm{n}=9)$ with a unique microbiome signature. Patients with PFI $<6$ months individuals had higher

308 odds $(\log \mathrm{OR}=1.85,95 \% \mathrm{CI}-0.85-4.497$, $\mathrm{p}$-value $=0.12)$ of being in this unique/outlier fecal

309 microbiome group. These fecal outliers have significantly lower phylogenetic diversity

310 compared to the other fecal samples ( $p$-value $=0.001$, Fig. $5 \mathrm{~A}$ ) and have increased abundance of

311 genera belonging to the order Clostridiales (Lachnospira [Kruskal Wallis p-value $=0.00039$ ],

312 unidentified Ruminococceae genus [Kruskal Wallis p-value $=0.001337$ ], and Subdoligranulum

313 Kruskal Wallis p-value $=0.01121])($ Fig. S7). Of the six platinum-resistant patients in this

314 outlier subset, two also had Escherichia-dominated vaginal communities. Eight of the nine 
315 patients in this subgroup reported consuming antibiotics within the past 6 months but this was

316 not a significantly greater proportion of individuals with recent antibiotic consumption compared

317 to individuals outside this fecal outlier subgroup $(\log \mathrm{OR}=0.35,95 \% \mathrm{CI}-1.44-3.05 \mathrm{p}$-value $=$

$3180.48)$.

319

320 Overall, patients with platinum-resistant disease had lower fecal phylogenetic diversity

321 compared to patients with platinum-sensitive disease, but the difference was not significant (Fig.

$3225 \mathrm{~B}$, p-value $=0.18$ ). Regardless of PFI, patients with ovarian cancer had significantly higher

323 relative abundance of Prevotella in the gut microbiome compared to benign individuals ( $\mathrm{p}$-value

$324=0.028$, Fig. S8). Outside of the above-mentioned associations, there were no other significant

325 associations between the gut microbiome and platinum sensitivity or other health/lifestyle

326 variables.

327

328 Discussion

329 One of the major findings of this study is the inverse relationship between a Lactobacillus-

330 dominant vaginal microbiome and ovarian cancer. In our study, fewer women than expected

331 (24.4\%) have Lactobacillus-dominated vaginal communities compared to similarly aged, healthy

332 women $(47.2 \%)$ from other studies (p-value $=0.037)(44,45)$. Lactobacillus dominance is not

333 found in any of the Black $(n=2)$ or Native American $(n=4)$ women in our study but only eleven

334 of the 39 white women have Lactobacillus-dominated vaginal microbiomes; therefore, ethnicity

335 was not a driving factor in Lactobacillus abundance in this study ( $\mathrm{p}$-value $=0.27$ ). These results

336 suggest the possibility that the low abundance of Lactobacillus may be indicative of a broader

337 relationship between ovarian cancer and the vaginal microbiome. This finding corroborates a 
338 previous study that also observed a reduced frequency of the Lactobacillus-dominated vaginal

339 microbiome in women with ovarian cancer, particularly in women under 50 (45). A partial

340 explanation may be that Lactobacillus-dominance, while typically viewed as a healthy state in

341 the female genital tract, is a non-resilient ecology prone to disruption by variable factors,

342 including changes in glycogen availability $(50,51)$, antibiotic exposure $(52)$, and shifts in

343 hormone abundance induced during stress responses (53). While we observed no statistical

344 relationships between Lactobacillus dominance and platinum-sensitivity or other

345 lifestyle/medical variables, there was a nearly significant decrease in Lactobacillus-dominance

346 associated with taking antibiotics within the past month ( $p$-value $=0.0515)$, as well as a positive

347 association between Lactobacillus-dominance and microbial cell density $\left(\mathrm{R}^{2}=0.278\right)$.

349 Lactobacillus maintains a low $\mathrm{pH}$ in the vaginal environment by producing lactic acid as a

350 byproduct of glycogen metabolism and this low $\mathrm{pH}$ inhibits growth of pro-inflammatory bacteria

351 (21, 22, 54). Vaginal Lactobacillus may protect from gynecological cancers by inhibiting pro-

352 inflammatory bacteria, such as those implicated in pelvic inflammatory disease, and by reducing 353 inflammatory cytokines IL-1 $\beta$ and IL-6 (55). The low Lactobacillus levels we observed may be 354 related to glycogen availability - nearly $75 \%$ of women in this study were post-menopause, and 355 vaginally produced glycogen is known to decrease after menopause; likewise, chemotherapy can 356 inhibit ovarian estrogen production and result in lower glycogen levels $(44,54,56,57)$. More

357 importantly, many women in this study have had at least one ovary surgically removed during 358 initial cancer treatment. Ovary removal leads to decreased estrogen production and thus, a likely 359 decrease in vaginal glycogen levels; however, more research is needed to fully explain the 360 ovarian-estrogen-glycogen dynamic (50). Glycogen abundance may also help explain the 
361 relationship between vaginal microbiome cell density and Lactobacillus, as widely available

362 glycogen may encourage a densely colonized Lactobacillus vaginal community due to high

363 nutrient availability (57). Hormone replacement therapy (HRT) can be used to replace estrogen

364 production that is stopped after menopause, and four women (two pre-menopause and two post-

365 menopause) were on HRT. Women on HRT or pre-menopause were more likely to have

366 Lactobacillus-dominated communities, but this was not significant $(\mathrm{p}$-value $=0.0744)$. While we

367 did not document glycogen levels in our study, the positive relationship between likely estrogen

368 presence and Lactobacillus abundance lends credence to the idea that the low proportion of

369 women with Lactobacillus abundance in our study is due to low estrogen, and thus glycogen.

370 The retrospective nature of our study means that we were unable to assess Lactobacillus levels in

371 women with ovarian cancer before they progressed to stage III/IV, or prior to chemotherapy.

372 Therefore, we could not investigate anti-gynecological cancer properties of vaginal

373 Lactobacillus; nevertheless, by comparing patients in this study to similarly aged women without

374 ovarian cancer, we present further evidence that low Lactobacillus levels are more common in

375 women with ovarian cancer $(p-v a l u e=0.037)$.

376

377 The presence, and size, of residual disease is strongly correlated with decreased survivability in

378 ovarian cancer (7) and therefore our finding that $L$. iners was at significantly higher abundance

$379(\mathrm{p}=0.0359)$ in patients with either no gross residual disease or residual disease $<1 \mathrm{~cm}$ after

380 treatment may point to $L$. iners as a potential path toward a biomarker. $L$. iners is a common

381 vaginal bacterium $(46,58,59)$ but its role in health and disease is sometimes contradictory $(21)$;

382 L. iners has been found at high relative abundance in low-grade squamous intraepithelial lesions

383 in the cervix but at low abundance in high-grade squamous intraepithelial lesions (26). Yet, 
384 another study found L. iners at high abundance in women with normal cytology when compared

385 to women with squamous intraepithelial lesions (60). Other studies have found L. iners to be

386 positively associated with cervical cancer $(61,62)$ but L. iners is also linked to clearance of HPV

387 (63), which is a causative agent of cervical cancer. Further research is necessary to better

388 understand the role of $L$. iners in gynecological cancers in general, and in the potential inhibition

389 of gross residual disease in ovarian cancer.

390

391 Lactobacillus is known to inhibit colonization and growth of Escherichia in the vaginal

392 microbiome $(64,65)$. The low abundance of Lactobacillus found in our study may present ample

393 opportunity for typically low abundance vaginal bacteria, such as Escherichia, to thrive and

394 proliferate in the absence of competition. Overgrowth of Escherichia only occurred in $17.8 \%$ of

395 patients in our study, yet $75 \%$ of those patients were in the PFI $<6$ months group; this finding

396 was statistically significant $(\mathrm{p}$-value $=0.024)$. Put another way, $35.3 \%$ of patients with PFI $<6$

397 months showed greater than 20\% relative abundance of Escherichia, as compared to $4.34 \%$ of

398 PFI $>24$ months and only one of 5 benign cases. The explanation for why Escherichia was

399 significantly more common in patients with platinum-resistant tumors is unclear, and because

400 this study was retrospective, we were unable to track Escherichia abundance before we knew

401 each patient's platinum-sensitivity. One possible pathway is via interactions between the

402 microbiome, immune system, and how platinum-based chemotherapies induce cancer cell death.

403 Platinum chemotherapies partially rely on ROS produced by host myeloid cells (15). Microbes

404 strongly influence immune system function, and hence, alteration in ROS production may be

405 more common in Escherichia-dominant vaginal microbiomes, which may render platinum-based

406 chemotherapies less effective, leading to platinum-resistance. Vaginal Escherichia may also 
407 cause an increased inflammatory response, such as during pelvic inflammatory disease (66), and

408 promote cancerous growth, resulting in a shortened PFI. The effect of Escherichia on platinum-

409 sensitivity warrants further investigation.

410

411 We also observed differences in the gut microbiome of women with platinum-resistant tumors,

412 compared to benign and platinum-sensitive. Similar to the prevalence of Escherichia-dominant

413 vaginal microbiomes, $35.3 \%$ of patients with PFI $<6$ months were fecal beta-diversity outliers

414 compared to the remainder of the fecal samples, while only $13.0 \%$ of PFI $>24$ and none of the

415 benign cases fell into this cluster $(p$-value $=0.12)$. Genera belonging to the Clostridiales order

416 (Subdoligranulum $(\mathrm{p}$-value $=0.01121)$ and Lachnospira $(\mathrm{p}$-value $=0.00039)$ were at higher

417 abundance in this group of outlier samples. Subdoligranulum, has been found at high abundance

418 in the stool of individuals with gastrointestinal neoplasms (67) and has a positive association

419 with blood-based markers of inflammation (68). Lachnospira has been found to be positively

420 correlated with a plant-based diet (69) and at high abundance in healthy controls in a study

421 investigating chronic kidney disease (70); yet, Lachnospira is also found at high abundance in

422 women with metabolic disorder and obesity (69). Additionally, we note lower phylogenetic

423 diversity in the gut microbiome of platinum-resistant tumors compared to both platinum-

424 sensitive and benign tumors ( $p$-value $=0.18$ ). Chemotherapy is a well-documented driver of

425 decreased gut microbiome alpha diversity (71-73); however, we did not observe a shift in alpha

426 diversity with time since last cycle of chemotherapy. High gut microbiome alpha diversity is

427 typically associated with improved human health $(74,75)$, although recent studies have started to

428 question this paradigm (76). Nevertheless, the unique gut microbiome beta diversity profile in a

429 subset of PFI $<6$ months, and the decreased alpha diversity in the full PFI $<6$ months population 
430 indicates that there may be long term relationship between platinum-resistance and the gut 431 microbiome.

432

433 Prevotella is enriched in both the platinum-resistant and platinum-sensitive study groups, 434 compared to the benign group ( $\mathrm{p}$-value $=0.028)$. Prevotella is typically only found at high 435 abundance in the gut microbiomes of non-industrial, traditional populations (77) and it is 436 associated with consumption of a plant-rich diet (77). Yet, some strains of Prevotella are found 437 in the gut microbiomes of industrial populations and are linked to pro-inflammatory states in the 438 gut microbiome $(78,79)$. Similar to decreased Lactobacillus in the vaginal microbiome of 439 patients with ovarian cancer, the relatively high abundance of Prevotella in the gut microbiome 440 of women with ovarian cancer indicates a notable shift in microbial composition. Studies with 441 larger control groups are necessary to address this relationship.

442

\section{Conclusions}

444 Our results demonstrate an association between the vaginal and gut microbiomes and platinum445 sensitivity in women with ovarian cancer. Escherichia-dominant vaginal communities are 446 significantly more likely to be present in patients with platinum-resistant tumors but the 447 explanatory mechanism for this relationship is currently unclear. Lab contamination and/or 448 collection methodology does not appear to play a role in vaginal Escherichia abundance, which 449 indicates that finding Escherichia at high relative abundance in patients with PFI $<6$ months is a 450 biological trend. 
452 We also observed shared vaginal and gut microbiome profiles in women with ovarian cancer,

453 with decreased dominance of Lactobacillus and increased relative abundance of Prevotella,

454 respectively, regardless of platinum-sensitivity. These results suggest shifts in microbiome

455 composition that are related to the ovarian cancer disease state, which may possibly be related to

456 chemotherapy, but the retrospective nature of our study does not allow us to distinguish the exact

457 mechanism of action.

458

459 Our results call for deeper investigation into the relationship between the vaginal and gut 460 microbiomes and ovarian cancer. A future avenue for research is a prospective, longitudinal

461 study that tracks how the vaginal and gut microbiomes change throughout the course of ovarian

462 cancer therapy, with an aim to disentangle how Escherichia-abundance impacts response to 463 chemotherapy. Similarly, a study tracking Lactobacillus abundance in an aged-matched,

464 lifestyle-matched cohort of women with and without ovarian cancer may provide insights into 465 how microbial risk factors impact occurrence and outcomes of ovarian cancer. This work must 466 also investigate why high $L$. iners abundance is found nearly exclusively in cases with $<1 \mathrm{~cm}$ or 467 no gross residual disease, while other Lactobacillus species are found in cases with $>1 \mathrm{~cm}$ 468 residual disease. Finally, ovarian cancer microbiome research also presents an opportunity for 469 microbial metagenomics and metabolomics to provide a fuller picture of the vaginal and gut 470 microbiome ecosystems in health and disease.

471

\section{Acknowledgements}


473 The authors would like to thank Sarah Cooper and Cathy Birdsong for their work in coordinating

474 patient recruitment and monitoring sample collection. The authors would also like to thank all

475 the individuals who participated in this study.

\section{Data Availability}

477 The 16S rRNA V4 sequencing data for each sample are available on NCBI under project ID

478 PRJNA662091. Bioinformatic scripts for processing the 16S rRNA V4 data are given in the

479 Supplementary Material.

480

481

482

\section{References}

483

484

485

486

487

488

489

490

491

492

493

494

495

496

497

498

499

500

501

502

503

504

505
1. Siegel RL, Miller KD, Jemal A. Cancer statistics, 2019. CA Cancer J Clin. 2019;69(1):7-34. Epub 2019/01/09. doi: 10.3322/caac.21551. PubMed PMID: 30620402.

2. Torre LA, Trabert B, DeSantis CE, Miller KD, Samimi G, Runowicz CD, Gaudet MM, Jemal A, Siegel RL. Ovarian cancer statistics, 2018. CA Cancer J Clin. 2018;68(4):284-96. Epub 2018/05/29. doi: 10.3322/caac.21456. PubMed PMID: 29809280; PMCID: PMC6621554.

3. Jacobs IJ, Menon U. Progress and challenges in screening for early detection of ovarian cancer. Mol Cell Proteomics. 2004;3(4):355-66. Epub 2004/02/07. doi: 10.1074/mcp.R400006MCP200. PubMed PMID: 14764655.

4. Baldwin LA, Huang B, Miller RW, Tucker T, Goodrich ST, Podzielinski I, DeSimone CP, Ueland FR, van Nagell JR, Seamon LG. Ten-year relative survival for epithelial ovarian cancer. Obstet Gynecol. 2012;120(3):612-8. Epub 2012/08/24. doi: 10.1097/AOG.0b013e318264f794. PubMed PMID: 22914471.

5. Raja FA, Chopra N, Ledermann JA. Optimal first-line treatment in ovarian cancer. Ann Oncol. 2012;23 Suppl 10(suppl_10):x118-27. Epub 2012/09/26. doi: 10.1093/annonc/mds315. PubMed PMID: 22987945.

6. Dasari S, Tchounwou PB. Cisplatin in cancer therapy: molecular mechanisms of action. European journal of pharmacology. 2014;740:364-78.

7. Chang S-J, Hodeib M, Chang J, Bristow RE. Survival impact of complete cytoreduction to no gross residual disease for advanced-stage ovarian cancer: a meta-analysis. Gynecologic oncology. 2013;130(3):493-8.

8. Ushijima K. Treatment for recurrent ovarian cancer-at first relapse. J Oncol. 2010;2010:497429. Epub 2010/01/13. doi: 10.1155/2010/497429. PubMed PMID: 20066162; PMCID: PMC2801501. 
506 9. Davis A, Tinker AV, Friedlander M. "Platinum resistant" ovarian cancer: what is it, who 507 to treat and how to measure benefit? Gynecologic oncology. 2014;133(3):624-31.

508 10. Bookman MA. Extending the platinum-free interval in recurrent ovarian cancer: the role 509 of topotecan in second-line chemotherapy. Oncologist. 1999;4(2):87-94. Epub 1999/05/25. 510 PubMed PMID: 10337378.

511 11. Gore M, Fryatt I, Wiltshaw E, Dawson T. Treatment of relapsed carcinoma of the ovary 512 with cisplatin or carboplatin following initial treatment with these compounds. Gynecologic 513 oncology. 1990;36(2):207-11.

514 12. Markman M, Rothman R, Hakes T, Reichman B, Hoskins W, Rubin S, Jones W, 515 Almadrones L, Lewis JL, Jr. Second-line platinum therapy in patients with ovarian cancer 516 previously treated with cisplatin. J Clin Oncol. 1991;9(3):389-93. Epub 1991/03/11. doi: 517 10.1200/JCO.1991.9.3.389. PubMed PMID: 1999708.

518 13. Pfisterer J, Ledermann JA, editors. Management of platinum-sensitive recurrent ovarian 519 cancer. Seminars in oncology; 2006: Elsevier.

520 14. Bossuet-Greif N, Vignard J, Taieb F, Mirey G, Dubois D, Petit C, Oswald E, Nougayrede JP. 521 The Colibactin Genotoxin Generates DNA Interstrand Cross-Links in Infected Cells. mBio.

522 2018;9(2):e02393-17. Epub 2018/03/22. doi: 10.1128/mBio.02393-17. PubMed PMID:

523 29559578; PMCID: PMC5874909.

524 15. lida N, Dzutsev A, Stewart CA, Smith L, Bouladoux N, Weingarten RA, Molina DA, Salcedo 525 R, Back T, Cramer S, Dai RM, Kiu H, Cardone M, Naik S, Patri AK, Wang E, Marincola FM, Frank 526 KM, Belkaid Y, Trinchieri G, Goldszmid RS. Commensal bacteria control cancer response to 527 therapy by modulating the tumor microenvironment. Science. 2013;342(6161):967-70. Epub 528 2013/11/23. doi: 10.1126/science.1240527. PubMed PMID: 24264989; PMCID: PMC6709532. 529 16. Perez-Chanona E, Trinchieri G. The role of microbiota in cancer therapy. Curr Opin 530 Immunol. 2016;39:75-81. Epub 2016/01/29. doi: 10.1016/j.coi.2016.01.003. PubMed PMID: 531 26820225; PMCID: PMC4801762.

532 17. Schwabe RF, Jobin C. The microbiome and cancer. Nat Rev Cancer. 2013;13(11):800-12. 533 Epub 2013/10/18. doi: 10.1038/nrc3610. PubMed PMID: 24132111; PMCID: PMC3986062.

534 18. Crusz SM, Balkwill FR. Inflammation and cancer: advances and new agents. Nat Rev Clin 535 Oncol. 2015;12(10):584-96. Epub 2015/07/01. doi: 10.1038/nrclinonc.2015.105. PubMed PMID: 53626122183.

537 19. Zackular JP, Baxter NT, Iverson KD, Sadler WD, Petrosino JF, Chen GY, Schloss PD. The 538 gut microbiome modulates colon tumorigenesis. mBio. 2013;4(6):e00692-13. Epub 2013/11/07. 539 doi: 10.1128/mBio.00692-13. PubMed PMID: 24194538; PMCID: PMC3892781.

540 20. Zackular JP, Rogers MA, Ruffin MT, Schloss PD. The human gut microbiome as a 541 screening tool for colorectal cancer. Cancer prevention research. 2014.

542 21. Champer M, Wong AM, Champer J, Brito IL, Messer PW, Hou JY, Wright JD. The role of 543 the vaginal microbiome in gynaecological cancer. BJOG. 2018;125(3):309-15. Epub 2017/03/10. 544 doi: 10.1111/1471-0528.14631. PubMed PMID: 28278350.

545 22. Chase D, Goulder A, Zenhausern F, Monk B, Herbst-Kralovetz M. The vaginal and 546 gastrointestinal microbiomes in gynecologic cancers: a review of applications in etiology, 547 symptoms and treatment. Gynecol Oncol. 2015;138(1):190-200. Epub 2015/05/10. doi: 548 10.1016/j.ygyno.2015.04.036. PubMed PMID: 25957158. 
549 23. Muls A, Andreyev J, Lalondrelle S, Taylor A, Norton C, Hart A. Systematic Review: The 550 Impact of Cancer Treatment on the Gut and Vaginal Microbiome in Women With a

551 Gynecological Malignancy. Int J Gynecol Cancer. 2017;27(7):1550-9. Epub 2017/06/08. doi:

552 10.1097/IGC.0000000000000999. PubMed PMID: 28590950; PMCID: PMC5571893.

553 24. Sharma H, Tal R, Clark NA, Segars JH, editors. Microbiota and pelvic inflammatory

554 disease. Seminars in reproductive medicine; 2014: Thieme Medical Publishers.

555 25. Lin HW, Tu YY, Lin SY, Su WJ, Lin WL, Lin WZ, Wu SC, Lai YL. Risk of ovarian cancer in 556 women with pelvic inflammatory disease: a population-based study. Lancet Oncol.

557 2011;12(9):900-4. Epub 2011/08/13. doi: 10.1016/S1470-2045(11)70165-6. PubMed PMID: 55821835693.

559 26. Xu J, Peng JJ, Yang W, Fu K, Zhang Y. Vaginal microbiomes and ovarian cancer: a review.

560 Am J Cancer Res. 2020;10(3):743-56. Epub 2020/04/09. PubMed PMID: 32266088; PMCID:

561 PMC7136922.

562 27. Shi N, Li N, Duan X, Niu H. Interaction between the gut microbiome and mucosal

563 immune system. Military Medical Research. 2017;4(1):1-7.

564 28. Thaiss CA, Zmora N, Levy M, Elinav E. The microbiome and innate immunity. Nature.

565 2016;535(7610):65-74. Epub 2016/07/08. doi: 10.1038/nature18847. PubMed PMID:

56627383981.

567 29. Ozben T. Oxidative stress and apoptosis: impact on cancer therapy. J Pharm Sci.

568 2007;96(9):2181-96. Epub 2007/06/27. doi: 10.1002/jps.20874. PubMed PMID: 17593552.

569 30. Mclnnes P, Cutting M. Manual of Procedures for Human Microbiome Project, Core

570 Microbiome Sampling, Protocol A, HMP Protocol \#07-001. 2010.

571 31. Caporaso JG, Lauber CL, Walters WA, Berg-Lyons D, Lozupone CA, Turnbaugh PJ, Fierer

572 N, Knight R. Global patterns of 16S rRNA diversity at a depth of millions of sequences per

573 sample. Proceedings of the national academy of sciences. 2011;108(Supplement 1):4516-22.

574 32. Schubert M, Lindgreen S, Orlando L. AdapterRemoval v2: rapid adapter trimming, 575 identification, and read merging. BMC Res Notes. 2016;9(1):88. Epub 2016/02/13. doi:

576 10.1186/s13104-016-1900-2. PubMed PMID: 26868221; PMCID: PMC4751634.

577 33. Caporaso JG, Kuczynski J, Stombaugh J, Bittinger K, Bushman FD, Costello EK, Fierer N,

578 Pena AG, Goodrich JK, Gordon JI, Huttley GA, Kelley ST, Knights D, Koenig JE, Ley RE, Lozupone 579 CA, McDonald D, Muegge BD, Pirrung M, Reeder J, Sevinsky JR, Turnbaugh PJ, Walters WA, 580 Widmann J, Yatsunenko T, Zaneveld J, Knight R. QIIME allows analysis of high-throughput 581 community sequencing data. Nat Methods. 2010;7(5):335-6. Epub 2010/04/13. doi:

582 10.1038/nmeth.f.303. PubMed PMID: $20383131 ;$ PMCID: PMC3156573.

583 34. Edgar RC. Search and clustering orders of magnitude faster than BLAST. Bioinformatics. 584 2010;26(19):2460-1.

585 35. Yoon SH, Ha SM, Kwon S, Lim J, Kim Y, Seo H, Chun J. Introducing EzBioCloud: a 586 taxonomically united database of 16S rRNA gene sequences and whole-genome assemblies. Int 587 J Syst Evol Microbiol. 2017;67(5):1613-7. Epub 2016/12/23. doi: 10.1099/ijsem.0.001755.

588 PubMed PMID: 28005526; PMCID: PMC5563544.

589 36. Lozupone C, Knight R. UniFrac: a new phylogenetic method for comparing microbial 590 communities. Appl Environ Microbiol. 2005;71(12):8228-35. Epub 2005/12/08. doi:

591 10.1128/AEM.71.12.8228-8235.2005. PubMed PMID: 16332807; PMCID: PMC1317376. 
592 37. Price MN, Dehal PS, Arkin AP. FastTree 2--approximately maximum-likelihood trees for 593 large alignments. PLoS One. 2010;5(3):e9490. Epub 2010/03/13. doi:

594 10.1371/journal.pone.0009490. PubMed PMID: 20224823; PMCID: PMC2835736.

595 38. Team RC. R: A language and environment for statistical computing2013.

596 39. Ward Jr JH. Hierarchical grouping to optimize an objective function. Journal of the

597 American statistical association. 1963;58(301):236-44.

598 40. Warnes MGR, Bolker B, Bonebakker L, Gentleman R, Huber W. Package 'gplots'. Various 599 R Programming Tools for Plotting Data. 2016.

600 41. Aragon T. epitools: Epidemiology Tools R package version 0.5-7. Berkeley, CA: University 601 of California. Retrieved from http://CRAN. R ...; 2012.

602 42. Wickham H. ggplot2: elegant graphics for data analysis: Springer; 2016.

603 43. Harrower M, Brewer CA. ColorBrewer. org: an online tool for selecting colour schemes 604 for maps. The Cartographic Journal. 2003;40(1):27-37.

605 44. Brotman RM, Shardell MD, Gajer P, Fadrosh D, Chang K, Silver MI, Viscidi RP, Burke AE, 606 Ravel J, Gravitt PE. Association between the vaginal microbiota, menopause status, and signs of 607 vulvovaginal atrophy. Menopause. 2014;21(5):450-8. Epub 2013/10/02. doi:

608 10.1097/GME.0b013e3182a4690b. PubMed PMID: 24080849; PMCID: PMC3994184.

609 45. Nené NR, Reisel D, Leimbach A, Franchi D, Jones A, Evans I, Knapp S, Ryan A, Ghazali S,

610 Timms JF. Association between the cervicovaginal microbiome, BRCA1 mutation status, and risk 611 of ovarian cancer: a case-control study. The Lancet Oncology. 2019;20(8):1171-82.

612 46. Ravel J, Gajer P, Abdo Z, Schneider GM, Koenig SS, McCulle SL, Karlebach S, Gorle R,

613 Russell J, Tacket CO, Brotman RM, Davis CC, Ault K, Peralta L, Forney LJ. Vaginal microbiome of 614 reproductive-age women. Proc Natl Acad Sci U S A. 2011;108 Suppl 1(Supplement 1):4680-7.

615 Epub 2010/06/11. doi: 10.1073/pnas.1002611107. PubMed PMID: 20534435; PMCID:

616 PMC3063603.

617 47. Lamont RF, Sobel JD, Akins RA, Hassan SS, Chaiworapongsa T, Kusanovic JP, Romero R.

618 The vaginal microbiome: new information about genital tract flora using molecular based 619 techniques. BJOG: An International Journal of Obstetrics \& Gynaecology. 2011;118(5):533-49.

620 48. Arumugam M, Raes J, Pelletier E, Le Paslier D, Yamada T, Mende DR, Fernandes GR, Tap 621 J, Bruls T, Batto JM, Bertalan M, Borruel N, Casellas F, Fernandez L, Gautier L, Hansen T, Hattori 622 M, Hayashi T, Kleerebezem M, Kurokawa K, Leclerc M, Levenez F, Manichanh C, Nielsen HB, 623 Nielsen T, Pons N, Poulain J, Qin J, Sicheritz-Ponten T, Tims S, Torrents D, Ugarte E, Zoetendal 624 EG, Wang J, Guarner F, Pedersen O, de Vos WM, Brunak S, Dore J, Meta HITC, Antolin M, 625 Artiguenave F, Blottiere HM, Almeida M, Brechot C, Cara C, Chervaux C, Cultrone A, Delorme C, 626 Denariaz G, Dervyn R, Foerstner KU, Friss C, van de Guchte M, Guedon E, Haimet F, Huber W, 627 van Hylckama-Vlieg J, Jamet A, Juste C, Kaci G, Knol J, Lakhdari O, Layec S, Le Roux K, Maguin E, 628 Merieux A, Melo Minardi R, M'Rini C, Muller J, Oozeer R, Parkhill J, Renault P, Rescigno M, 629 Sanchez N, Sunagawa S, Torrejon A, Turner K, Vandemeulebrouck G, Varela E, Winogradsky Y, 630 Zeller G, Weissenbach J, Ehrlich SD, Bork P. Enterotypes of the human gut microbiome. Nature. 631 2011;473(7346):174-80. Epub 2011/04/22. doi: 10.1038/nature09944. PubMed PMID:

$63221508958 ;$ PMCID: PMC3728647.

633 49. Schloissnig S, Arumugam M, Sunagawa S, Mitreva M, Tap J, Zhu A, Waller A, Mende DR, 634 Kultima JR, Martin J, Kota K, Sunyaev SR, Weinstock GM, Bork P. Genomic variation landscape 
635 of the human gut microbiome. Nature. 2013;493(7430):45-50. Epub 2012/12/12. doi:

636 10.1038/nature11711. PubMed PMID: $23222524 ;$ PMCID: PMC3536929.

637 50. Mirmonsef P, Hotton AL, Gilbert D, Burgad D, Landay A, Weber KM, Cohen M, Ravel J,

638 Spear GT. Free glycogen in vaginal fluids is associated with Lactobacillus colonization and low 639 vaginal pH. PloS one. 2014;9(7):e102467.

640 51. Mirmonsef P, Modur S, Burgad D, Gilbert D, Golub ET, French AL, McCotter K, Landay AL, 641 Spear GT. An exploratory comparison of vaginal glycogen and Lactobacillus levels in pre-and 642 post-menopausal women. Menopause (New York, NY). 2015;22(7):702.

643 52. Melkumyan A, Priputnevich T, Ankirskaya A, Murav'eva V, Lubasovskaya L. Effects of 644 antibiotic treatment on the lactobacillus composition of vaginal microbiota. Bulletin of 645 experimental biology and medicine. 2015;158(6):766-8.

646 53. Witkin SS, Linhares IM. Why do lactobacilli dominate the human vaginal microbiota? 647 BJOG: An International Journal of Obstetrics \& Gynaecology. 2017;124(4):606-11.

648 54. Mirmonsef P, Hotton AL, Gilbert D, Gioia CJ, Maric D, Hope TJ, Landay AL, Spear GT. 649 Glycogen levels in undiluted genital fluid and their relationship to vaginal $\mathrm{pH}$, estrogen, and 650 progesterone. PloS one. 2016;11(4):e0153553.

651 55. Hemalatha R, Mastromarino P, Ramalaxmi BA, Balakrishna NV, Sesikeran B.

652 Effectiveness of vaginal tablets containing lactobacilli versus $\mathrm{pH}$ tablets on vaginal health and 653 inflammatory cytokines: a randomized, double-blind study. Eur J Clin Microbiol Infect Dis.

654 2012;31(11):3097-105. Epub 2012/07/11. doi: 10.1007/s10096-012-1671-1. PubMed PMID:

65522777592.

656 56. Bachmann GA, Nevadunsky NS. Diagnosis and treatment of atrophic vaginitis. Am Fam 657 Physician. 2000;61(10):3090-6. Epub 2000/06/06. PubMed PMID: 10839558.

658 57. Muhleisen AL, Herbst-Kralovetz MM. Menopause and the vaginal microbiome. 659 Maturitas. 2016;91:42-50. Epub 2016/07/28. doi: 10.1016/j.maturitas.2016.05.015. PubMed 660 PMID: 27451320.

661 58. Lewis FM, Bernstein KT, Aral SO. Vaginal Microbiome and Its Relationship to Behavior, 662 Sexual Health, and Sexually Transmitted Diseases. Obstet Gynecol. 2017;129(4):643-54. Epub 663 2017/03/10. doi: 10.1097/AOG.0000000000001932. PubMed PMID: 28277350; PMCID:

664 PMC6743080.

665 59. Younes JA, Lievens E, Hummelen R, van der Westen R, Reid G, Petrova MI. Women and 666 their microbes: the unexpected friendship. Trends in microbiology. 2017.

667 60. Audirac-Chalifour A, Torres-Poveda K, Bahena-Román M, Téllez-Sosa J, Martínez-

668 Barnetche J, Cortina-Ceballos B, López-Estrada G, Delgado-Romero K, Burguete-García Al, Cantú

669 D. Cervical microbiome and cytokine profile at various stages of cervical cancer: a pilot study.

670 PloS one. 2016;11(4):e0153274.

671 61. Oh HY, Kim BS, Seo SS, Kong JS, Lee JK, Park SY, Hong KM, Kim HK, Kim MK. The

672 association of uterine cervical microbiota with an increased risk for cervical intraepithelial

673 neoplasia in Korea. Clin Microbiol Infect. 2015;21(7):674 e1-9. Epub 2015/03/11. doi:

674 10.1016/j.cmi.2015.02.026. PubMed PMID: 25752224.

675 62. Seo SS, Oh HY, Lee JK, Kong JS, Lee DO, Kim MK. Combined effect of diet and cervical

676 microbiome on the risk of cervical intraepithelial neoplasia. Clin Nutr. 2016;35(6):1434-41. Epub

677 2016/04/15. doi: 10.1016/j.clnu.2016.03.019. PubMed PMID: 27075319. 
678 63. Brotman RM, Shardell MD, Gajer P, Tracy JK, Zenilman JM, Ravel J, Gravitt PE. Interplay 679 between the temporal dynamics of the vaginal microbiota and human papillomavirus detection. 680 The Journal of infectious diseases. 2014;210(11):1723-33.

681 64. Delley M, Bruttin A, Richard M, Affolter M, Rezzonico E, Bruck WM. In vitro activity of 682 commercial probiotic Lactobacillus strains against uropathogenic Escherichia coli. FEMS 683 Microbiol Lett. 2015;362(13):fnv096. Epub 2015/06/17. doi: 10.1093/femsle/fnv096. PubMed 684 PMID: 26078118.

685 65. Gupta K, Stapleton AE, Hooton TM, Roberts PL, Fennell CL, Stamm WE. Inverse 686 association of $\mathrm{H} 2 \mathrm{O} 2$-producing lactobacilli and vaginal Escherichia coli colonization in women 687 with recurrent urinary tract infections. J Infect Dis. 1998;178(2):446-50. Epub 1998/08/11. doi: 688 10.1086/515635. PubMed PMID: 9697725.

689 66. Heinonen PK, Miettinen A. Laparoscopic study on the microbiology and severity of acute 690 pelvic inflammatory disease. Eur J Obstet Gynecol Reprod Biol. 1994;57(2):85-9. Epub 691 1994/11/01. doi: 10.1016/0028-2243(94)90048-5. PubMed PMID: 7859910.

692 67. Youssef O, Lahti L, Kokkola A, Karla T, Tikkanen M, Ehsan H, Carpelan-Holmstrom M, 693 Koskensalo S, Bohling T, Rautelin H, Puolakkainen P, Knuutila S, Sarhadi V. Stool Microbiota 694 Composition Differs in Patients with Stomach, Colon, and Rectal Neoplasms. Dig Dis Sci. 695 2018;63(11):2950-8. Epub 2018/07/12. doi: 10.1007/s10620-018-5190-5. PubMed PMID: 696 29995183; PMCID: PMC6182444.

697 68. de Groot PF, Belzer C, Aydin O, Levin E, Levels JH, Aalvink S, Boot F, Holleman F, van 698 Raalte DH, Scheithauer TP, Simsek S, Schaap FG, Olde Damink SWM, Roep BO, Hoekstra JB, de 699 Vos WM, Nieuwdorp M. Distinct fecal and oral microbiota composition in human type 1 700 diabetes, an observational study. PLoS One. 2017;12(12):e0188475. Epub 2017/12/07. doi: 701 10.1371/journal.pone.0188475. PubMed PMID: 29211757; PMCID: PMC5718513.

702 69. Vacca M, Celano G, Calabrese FM, Portincasa P, Gobbetti M, De Angelis M. The 703 Controversial Role of Human Gut Lachnospiraceae. Microorganisms. 2020;8(4):573. Epub 704 2020/04/25. doi: 10.3390/microorganisms8040573. PubMed PMID: 32326636; PMCID: 705 PMC7232163.

706 70. Lun H, Yang W, Zhao S, Jiang M, Xu M, Liu F, Wang Y. Altered gut microbiota and 707 microbial biomarkers associated with chronic kidney disease. Microbiologyopen. 708 2019;8(4):e00678. Epub 2018/08/09. doi: 10.1002/mbo3.678. PubMed PMID: 30088332; 709 PMCID: PMC6460263.

710 71. Montassier E, Gastinne T, Vangay P, Al-Ghalith GA, Bruley des Varannes S, Massart S, 711 Moreau P, Potel G, de La Cochetiere MF, Batard E, Knights D. Chemotherapy-driven dysbiosis in 712 the intestinal microbiome. Aliment Pharmacol Ther. 2015;42(5):515-28. Epub 2015/07/07. doi: 713 10.1111/apt.13302. PubMed PMID: 26147207.

714 72. Hakim H, Dallas R, Wolf J, Tang L, Schultz-Cherry S, Darling V, Johnson C, Karlsson EA, 715 Chang TC, Jeha S, Pui CH, Sun Y, Pounds S, Hayden RT, Tuomanen E, Rosch JW. Gut Microbiome 716 Composition Predicts Infection Risk During Chemotherapy in Children With Acute

717 Lymphoblastic Leukemia. Clin Infect Dis. 2018;67(4):541-8. Epub 2018/03/09. doi:

718 10.1093/cid/ciy153. PubMed PMID: 29518185; PMCID: PMC6070042.

719 73. Alexander JL, Wilson ID, Teare J, Marchesi JR, Nicholson JK, Kinross JM. Gut microbiota 720 modulation of chemotherapy efficacy and toxicity. Nat Rev Gastroenterol Hepatol. 
721 2017;14(6):356-65. Epub 2017/03/09. doi: 10.1038/nrgastro.2017.20. PubMed PMID:

72228270698.

723 74. Menni C, Jackson MA, Pallister T, Steves CJ, Spector TD, Valdes AM. Gut microbiome

724 diversity and high-fibre intake are related to lower long-term weight gain. Int J Obes (Lond).

725 2017;41(7):1099-105. Epub 2017/03/14. doi: 10.1038/ijo.2017.66. PubMed PMID: 28286339;

726 PMCID: PMC5500185.

727 75. Turnbaugh PJ, Hamady M, Yatsunenko T, Cantarel BL, Duncan A, Ley RE, Sogin ML, Jones

728 WJ, Roe BA, Affourtit JP, Egholm M, Henrissat B, Heath AC, Knight R, Gordon JI. A core gut

729 microbiome in obese and lean twins. Nature. 2009;457(7228):480-4. Epub 2008/12/02. doi:

730 10.1038/nature07540. PubMed PMID: 19043404; PMCID: PMC2677729.

731 76. Reese AT, Dunn RR. Drivers of Microbiome Biodiversity: A Review of General Rules,

732 Feces, and Ignorance. mBio. 2018;9(4):e01294-18. Epub 2018/08/02. doi: 10.1128/mBio.01294-

733 18. PubMed PMID: $30065092 ;$ PMCID: PMC6069118.

$73477 . \quad$ Ley RE. Gut microbiota in 2015: Prevotella in the gut: choose carefully. Nat Rev

735 Gastroenterol Hepatol. 2016;13(2):69-70. Epub 2016/02/02. doi: 10.1038/nrgastro.2016.4.

736 PubMed PMID: 26828918.

737 78. Larsen JM. The immune response to Prevotella bacteria in chronic inflammatory disease.

738 Immunology. 2017;151(4):363-74. Epub 2017/05/26. doi: 10.1111/imm.12760. PubMed PMID:

739 28542929; PMCID: PMC5506432.

$74079 . \quad$ Scher JU, Sczesnak A, Longman RS, Segata N, Ubeda C, Bielski C, Rostron T, Cerundolo V,

741 Pamer EG, Abramson SB, Huttenhower C, Littman DR. Expansion of intestinal Prevotella copri

742 correlates with enhanced susceptibility to arthritis. Elife. 2013;2:e01202. Epub 2013/11/07. doi:

743 10.7554/eLife.01202. PubMed PMID: 24192039; PMCID: PMC3816614.

744 80. Fang X, Monk JM, Nurk S, Akseshina M, Zhu Q, Gemmell C, Gianetto-Hill C, Leung N,

745 Szubin R, Sanders J. Metagenomics-based, strain-level analysis of Escherichia coli from a time-

746 series of microbiome samples from a Crohn's disease patient. Frontiers in microbiology.

747 2018;9:2559.

748 81. Gao Y-D, Zhao Y, Huang J. Metabolic modeling of common Escherichia coli strains in

749 human gut microbiome. BioMed research international. 2014;2014.

750 82. Million M, Thuny F, Angelakis E, Casalta J, Giorgi R, Habib G, Raoult D. Lactobacillus

751 reuteri and Escherichia coli in the human gut microbiota may predict weight gain associated

752 with vancomycin treatment. Nutrition \& diabetes. 2013;3(9):e87-e.

753 


\section{Table $\mathbf{1}$ (on next page)}

Table 1: Study Group Demographics

Number of individuals belonging to each study group per clinical/lifestyle variable. The percent of individuals within each study category belonging to each metadata variable are presented in parentheses. Metadata variables that are significantly different between study groups are represented by ${ }^{\circledR}$ and Table S1 presents the p-value for each metadata variable. 


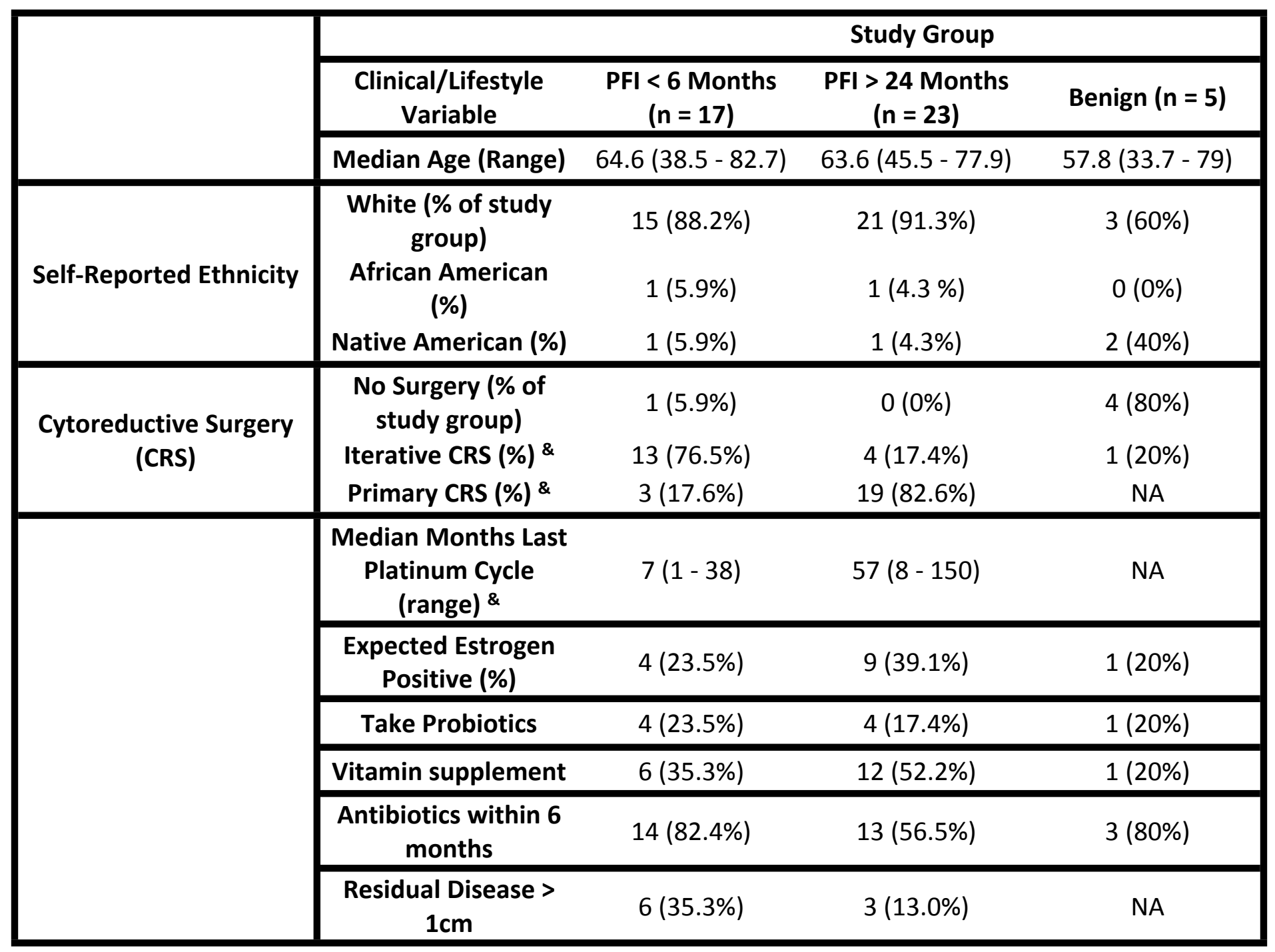

1

2

3

4

5 


\section{Figure 1}

Figure 1: Heatmap of the 25 most abundant genera in the vaginal microbiome.

Each column represents a single individual's vaginal microbiome, color coded by study group. Colors of each cell are based on a heatmap, ranging from 0 reads of the bacteria in that individual (white) to 9000 reads of the bacteria in that individual (red). Samples were rarefied to 9000 reads, so bright red indicates every read in the sample comes from that bacteria. Samples were clustered together based on similarity of vaginal microbiome using Ward hierarchical clustering. 11 microbiomes were Lactobacillus-dominated, six Escherichiadominated, three Gardnerella-dominated, nine Prevotella, and 16 highly diverse. 


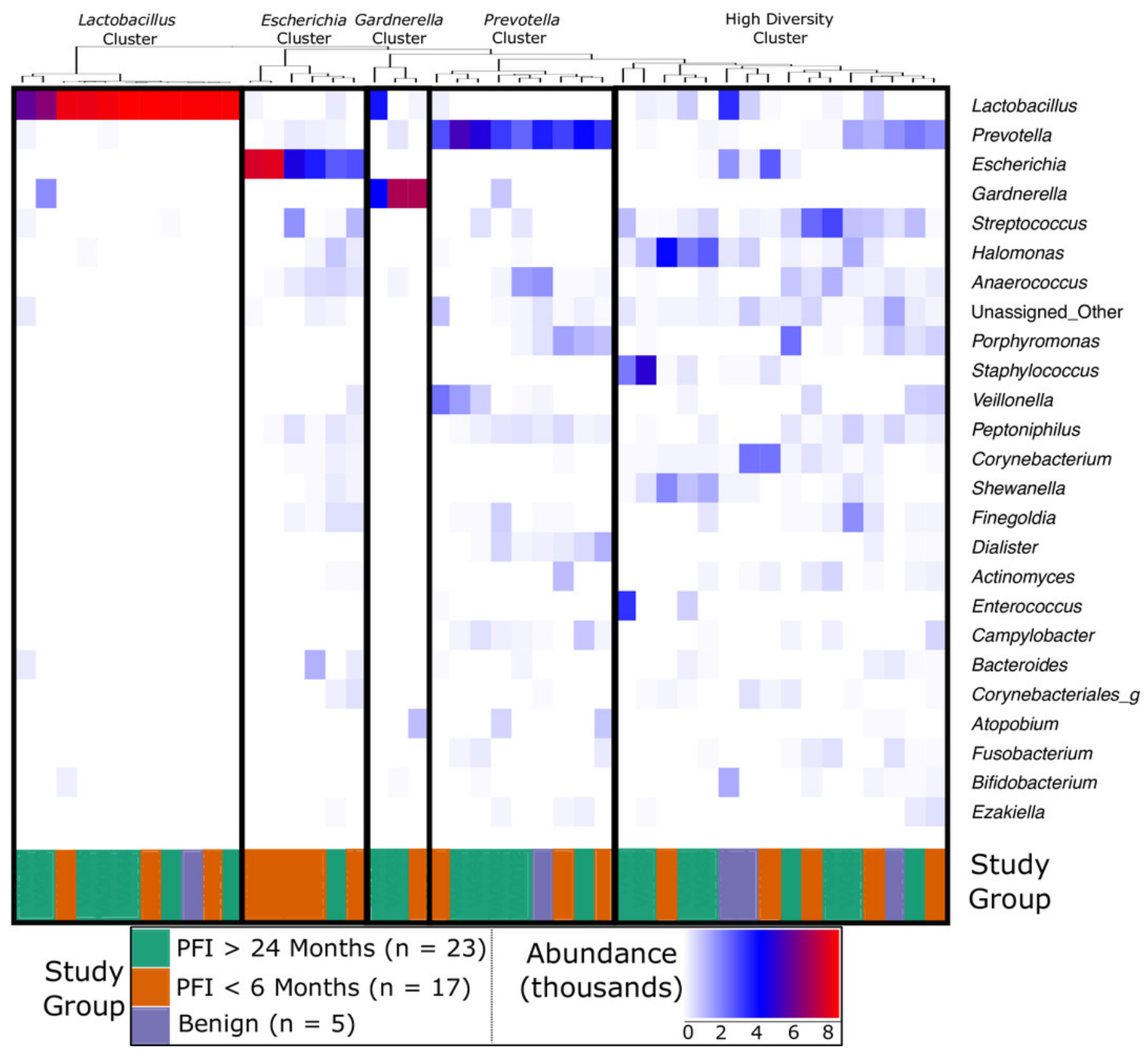




\section{Figure 2}

Figure 2A-D: Log-transformed odds ratio vaginal microbiome dominance.

Log-transformed odds are represented by the orange circle and the bars represent 95\% confidence intervals. P-values are given for each odds ratio and a significant result is indicated when the $95 \%$ confidence interval is completely greater than 0 or completely less than 0. A) Escherichia-dominance had significantly higher odds of occurring in PFI $<6$ months individuals ( $p$-value $=0.024$ ). B) Antibiotics within one month was negatively associated with Lactobacillus-dominance, but not significantly ( $p$-value $=0.051$ ). There were no differences between medical/health/lifestyle variables in Prevotella (C) and highly diverse (D) vaginal microbiomes. Gardnerella-dominated communities were not included in this analysis due to small sample size $(n=3)$. 

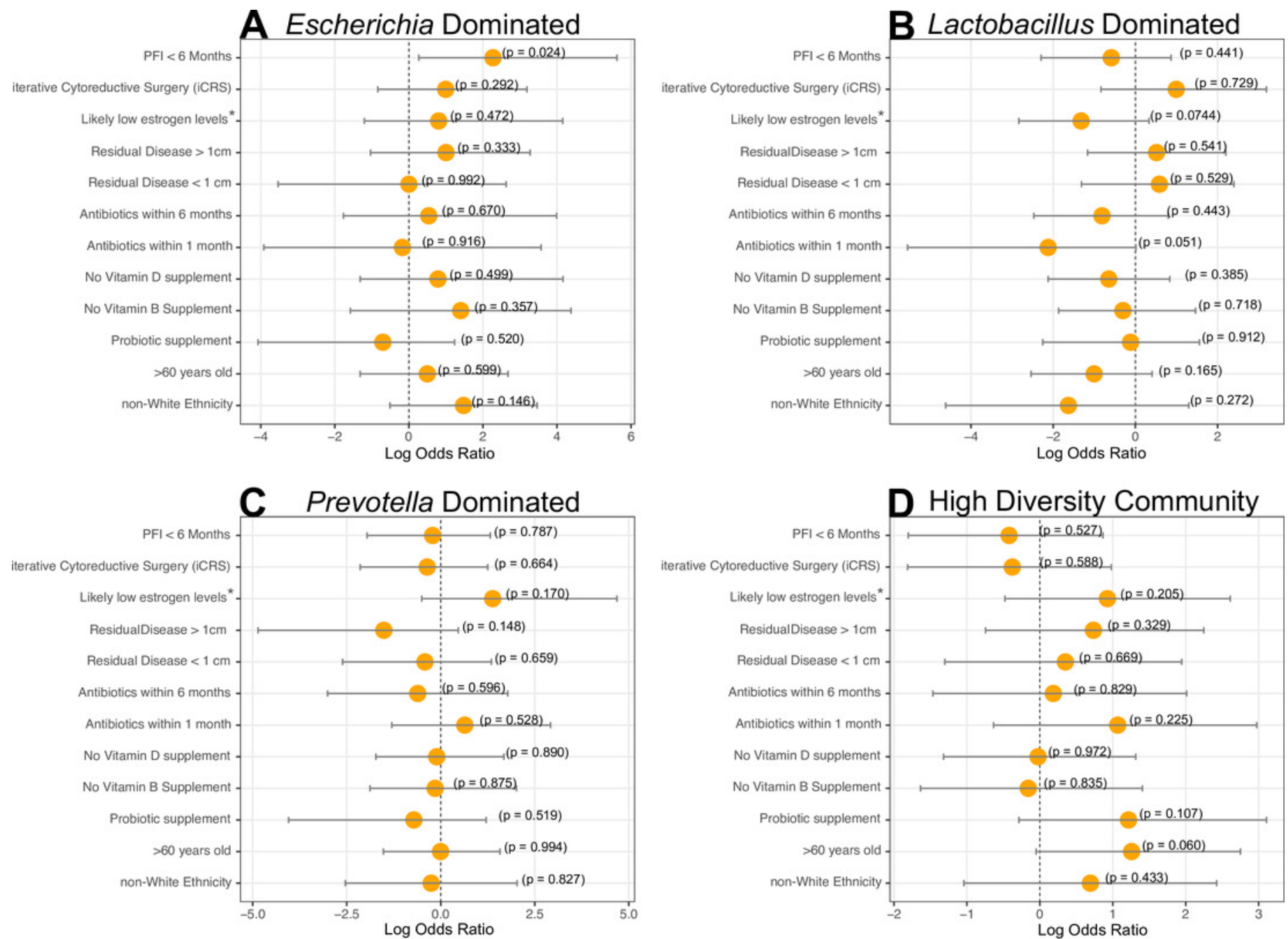

* Postmenopausal women not on hormone replacement therapy were classified as having likely low estrogen levels 


\section{Figure 3}

Figure 3: Lactobacillus iners dominates in small gross residual disease.

In individuals with Lactobacillus dominated vaginal microbiomes $(n=11), L$. iners was at significantly higher relative abundance $(p$-value $=0.0359)$ in patients with no gross residual disease or residual disease under $1 \mathrm{~cm}$. 


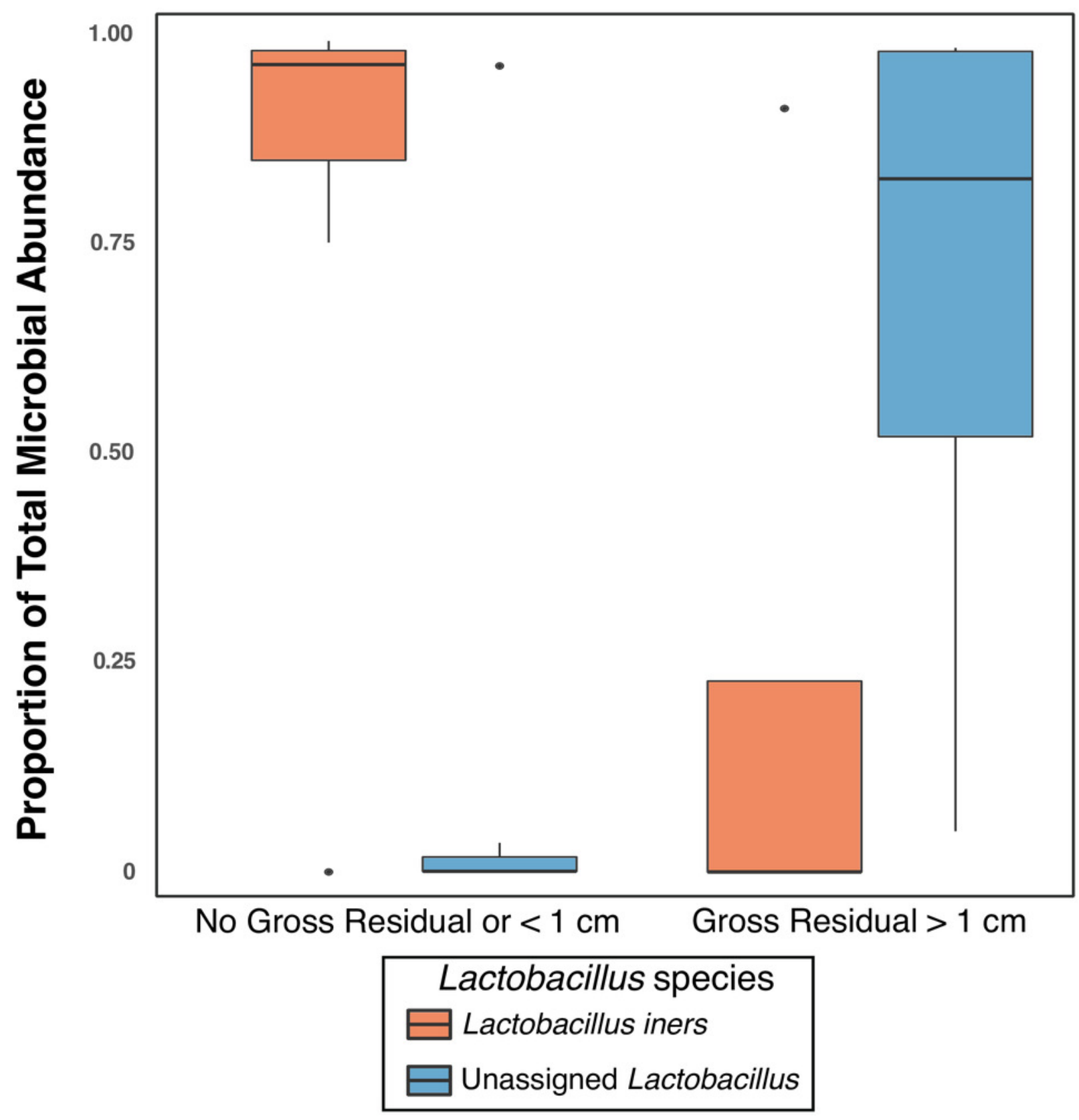


Figure 4

Figure 4: Unweighted UniFrac distances (PC1 and PC2) of fecal microbiomes from women with ovarian cancer.

Each shape represents a single sample and shapes clustering together have similar gut microbiome taxonomic composition. There was no significant difference in overall microbiome community structure between sample groups (PERMANOVA p-value $>0.05$ ); however, there are nine samples (6 PFI $<6$ months, 3 PFI $>24$ months) that form an outlier group along PC1. These individuals are labelled.

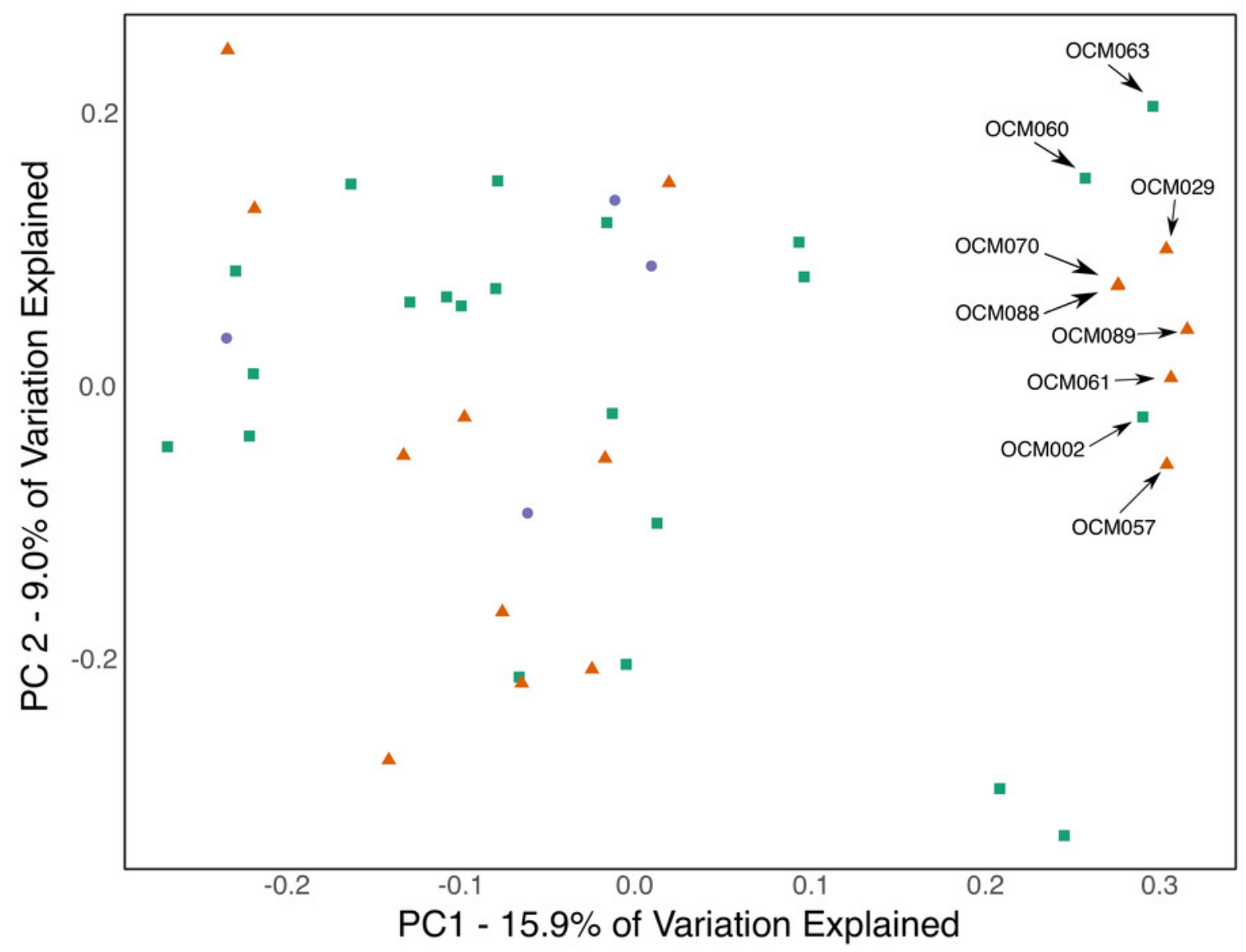

$\mathrm{PFI}<6$ Months $\mathrm{PFI}>24$ Months Benign Cases 


\section{Figure 5}

Figure 5A-B: Phylogenetic diversity in fecal microbiomes.

A) Samples that formed the outlier group in Figure $4(n=9)$ had lower phylogenetic diversity compared to the remainder of the gut microbiome samples ( $p$-value $=0.0001)$. B) Patients with PFI $<6$ months had lower phylogenetic diversity than benign and platinum-sensitive patients but this was not a significant result ( $p$-value $=0.18$ ).
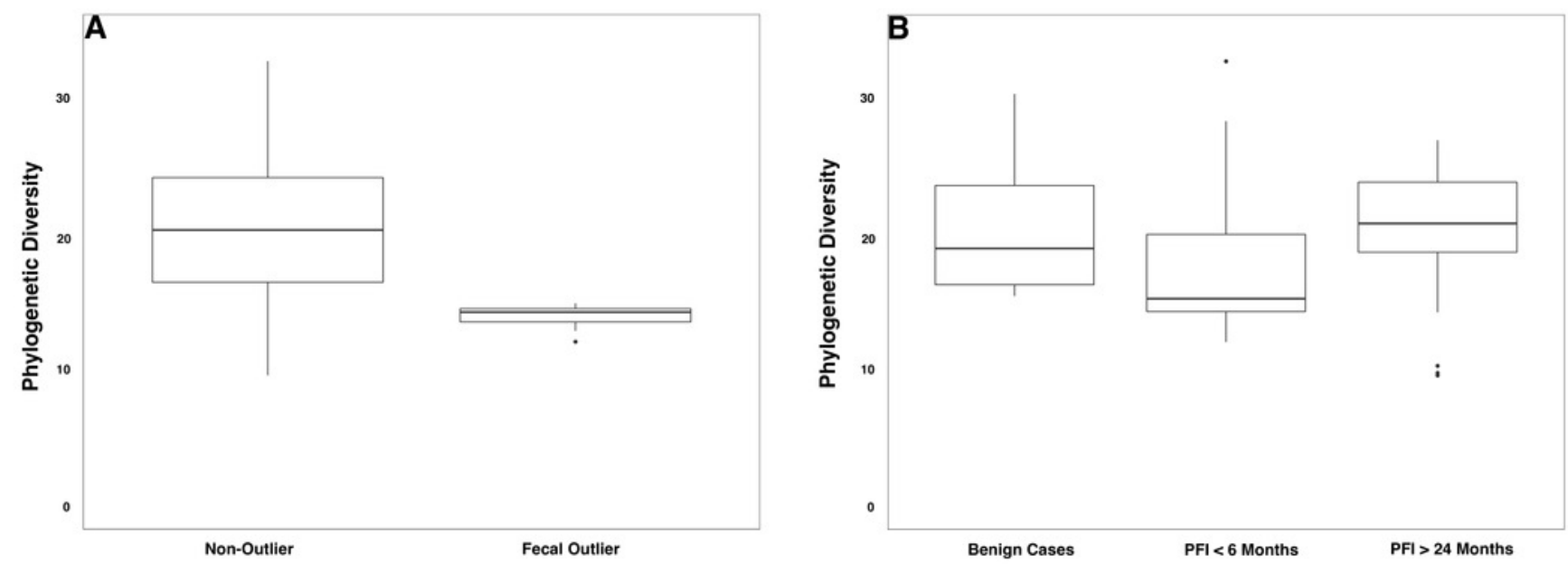